\title{
Exploring Multicriteria Decision Strategies in GIS with Linguistic Quantifiers: A Case Study of Residential Quality Evaluation
}

\author{
Jacek Malczewski
}

University of Western Ontario

\section{Claus Rinner}

University of Toronto

Malczewski, J., \& Rinner, C. (2005). Exploring multicriteria decision strategies in GIS with linguistic quantifiers: A case study of residential quality evaluation. Journal of Geographical Systems, 7(2), 249-268.

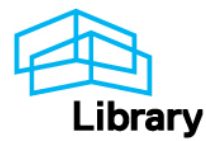




\title{
ORIGINAL PAPER
}

Jacek Malczewski · Claus Rinner

\section{Exploring multicriteria decision strategies in GIS with linguistic quantifiers: A case study of residential quality evaluation}

Received: 16 December 2003/ Accepted: 27 August 2004

(C) Springer-Verlag 2005

\begin{abstract}
Commonly used GIS combination operators such as Boolean conjunction/disjunction and weighted linear combination can be generalized to the ordered weighted averaging (OWA) family of operators. This multicriteria evaluation method allows decision-makers to define a decision strategy on a continuum between pessimistic and optimistic strategies. Recently, OWA has been introduced to GIS-based decision support systems. We propose to extend a previous implementation of OWA with linguistic quantifiers to simplify the definition of decision strategies and to facilitate an exploratory analysis of multiple criteria. The linguistic quantifier-guided OWA procedure is illustrated using a dataset for evaluating residential quality of neighborhoods in London, Ontario.
\end{abstract}

Keywords GIS · Multicriteria decision analysis · Ordered weighted averaging (OWA) $\cdot$ Linguistic quantifiers $\cdot$ Decision strategy $\cdot$ Residential quality evaluation

JEL Classification C61 $\cdot$ D89 $\cdot$ R29 $\cdot$ Z00

We would like to thank the anonymous reviewers for their constructive comments on the manuscript

\footnotetext{
J. Malczewski $(\square)$

Department of Geography, University of Western Ontario,

London, ON, N6A 5C2 Canada

E-mail: jmalczew@uwo.ca

C. Rinner

Department of Geography, University of Toronto,

100 Saint George Street, Toronto, ON, M5S 3G3 Canada

E-mail: rinner@geog.utoronto.ca
} 


\section{Introduction}

There is now a well-established body of literature on multicriteria evaluation and decision analysis in GIS (Janssen and Rietveld 1990; Carver 1991; Church et al. 1992; Banai 1993; Pereira and Duckstein 1993; Jankowski 1995; Laaribi et al. 1996; Eastman 1997; Malczewski 1999; Thill 1999; Joerin et al. 2001). Central to the multicriteria procedures is the concept of decision (or combination) rule. A decision rule dictates how best to order alternatives or to decide which alternative is preferred to another (Starr and Zeleny 1977). In the context of GIS applications, a decision rule specifies how to combine a set of criterion maps so that alternative decisions (locations) can be ordered according to some preferences with respect to evaluation criteria. Simple additive weighting (SAW) or weighted linear combination (WLC) and the Boolean operators are the most often used decision rules in GIS (Janssen and Rietveld 1990; Eastman 1997; Heywood et al. 2002; O'Sullivan and Unwin 2003). It can be shown that these rules belong to the family of ordered weighted averaging (OWA) operators (Yager 1988).

OWA involves a set of order weights that define a particular behavior of the operator in two dimensions: the degree of ORness (risk) and the tradeoff between criteria (Yager 1988; Jiang and Eastman 2000). These two dimensions form a decision strategy space (Jiang and Eastman 2000; Rinner and Malczewski 2002). By changing the order weights one can explore the decision space along these two dimensions. The decision space contains three special cases corresponding to the WLC and Boolean (AND and OR) combination rules. Jiang and Eastman (2000) describe which decision strategies in GIS can be realized by different OWA parameterizations. This perspective is based on Yager's (1988) observation that the AND-type combination can be associated with a pessimistic or risk-averse approach to decision-making while the OR-type combination represents an optimistic or risk-taking strategy.

Like many GIS applications, the research on integrating GIS and multicriteria analysis has recently been influenced by the development of the Internet (Carver 1999; Zhu and Dale 2001; Rinner and Malczewski 2002; Rinner 2003). This development has coincided with significant advancements in visualization methods that have led to a focus on exploratory approaches to multicriteria decision analysis (Heywood et al. 1995; Jankowski et al. 2001). Heywood et al. (1995) suggest that GISbased multicriteria analysis should involve a comparison of results obtained by using different decision rules. Jankowski et al. (2001) emphasize the importance of exploratory decision analysis in their implementation of multicriteria models in DECADE/CommonGIS (see also Andrienko and Andrienko 1999; Voß et al. 2000; Andrienko and Andrienko 2003). Rinner and Malczewski (2002) extend the decision support capabilities of CommonGIS by adding an OWA module. The module represents a conventional (quantitative) approach to the OWA operators. However, there is some evidence to suggest that the conventional OWA procedures have limited applicability in situations involving a large set of evaluation criteria (Yager and Kacprzyk 1997). In this case, it is difficult to combine 
the many criteria in a way that reflects the decision maker's presumed relationship between the criteria (Nijkamp et al. 1985; Malczewski et al. 2003). In such situations, the key aspects of the decision problem are often specified in terms of some linguistic (fuzzy) quantifiers such as at least $50 \%$ of the criteria must be satisfied or all the criteria must be satisfied, etc. (Zadeh 1983). This calls for an extension of the conventional OWA so that it can accommodate situations involving qualitative statements in the form of linguistic or fuzzy quantifiers (Yager 1988).

The problem of measuring quality of life (or residential quality) provides a good example of a situation in which one has to combine a large number of evaluation criteria (indicators) to obtain a composite measure of the quality of life. It typically involves a set of indicators on socioeconomic conditions of residents, the quality of the physical environment, accessibility to public and private sector services, etc. (Raphael 1996; Massam 2002). The different indicators are then combined to obtain an overall measure of the quality of life. Many studies rely on multivariate statistics and/or multicriteria evaluation procedures (Bayless and Bayless 1982; Can 1992; Raphael 1996; CUISR 2000). The simple additive weighting is the most often used approach for obtaining a composite measure of quality of life (e.g. Raphael 1996; CUISR 2000; Massam 2002). It is important to notice that there are two strong assumptions implicit in the SAW method: the linearity and additivity of criteria (Hwang and Yoon 1981; Malczewski 2000). The former assumption means that the desirability of an additional unit of a criterion is constant for any level of that criterion. The additivity assumption implies that the attributes under consideration are mutually preference-independent of each other. Arguably, these two assumptions are often difficult to apply in spatial decision-making/evaluation problems (Malczewski 2000). While some researchers contest the legitimacy of using SAW as a composite measure of quality of life (Bayless and Bayless 1982; Can 1992; Sharpe 1999), it has been argued that "theory, simulation, computations, and experience all suggest that the SAW method yields extremely close approximations to very much more complicated non-linear forms, while remaining far easier to use and understand" (Hwang and Yoon 1981, p. 103; see also Massam 1993). There is also empirical evidence to show that the method is "remarkably accurate in predicting individual judgments in both laboratory and applied setting" and that it is "robust to deviation from the underlying assumptions" (Hogarth 1983, p. 56).

There are two objectives of this paper: (1) to adapt the linguistic quantifier-based OWA approach to the GIS environment, and (2) to demonstrate how the approach can be used for evaluating residential quality. In the following section, we will define OWA and briefly review the features of the Web-enabled OWA implementation in the CommonGIS software. Section 3 provides an introduction to the concept of linguistic quantifiers and presents a quantifier-based extension of OWA in CommonGIS. In Sect. 4, we demonstrate an exploratory analysis of decision (or evaluation) strategies using linguistic quantifiers and Canadian census data for evaluating residential quality in London, Ontario. The final section presents concluding remarks. 


\section{Ordered weighted averaging in GIS}

\subsection{OWA and decision strategies}

Yager (1988) proposed OWA as a parameterized family of combination operators. Given a set of $n$ weighted standardized criterion (attribute) values $\left(w_{1} a_{i 1}, w_{2} a_{i 2}, \ldots, w_{n} a_{i n}\right)$ for each alternative $i$, and a set of order weights $\left(v_{1}\right.$, $v_{2}, \ldots, v_{n}, 0 \leq v_{j} \leq 1$, and $\left.\sum_{j=1}^{n} v_{j}=1\right)$, OWA can be defined as follows:

$$
O W A_{i}=\sum_{j=1}^{n} v_{j} b_{i j}
$$

where $b_{i 1} \geq b_{i 2} \geq \cdots \geq b_{i n}$ is the sequence obtained by reordering the weighted criterion values, $w_{1} a_{i 1}, w_{2} a_{i 2}, \ldots, w_{n} a_{i n}$ (Rinner and Malczewski 2002). According to the standard assumptions behind multicriteria decision analysis the criterion weights have the following properties: $0 \leq w_{j} \leq 1, \sum_{j=1}^{n} w_{j}=1$, and the attribute values are standardized so that $0 \leq a_{i j} \leq 1$ for the $i$-th alternative $(i=1,2, \ldots, m)$ and the $j$-th attribute $(j=1,2, \ldots, n)$ (see e.g. Carver 1991; Pereira and Duckstein 1993; Eastman 1997; Malczewski 1999).

The computation of OWA in the GIS environment involves the following steps: (1) define order weights, (2) sort the weighted standardized criterion values of each alternative (location) in descending order, (3) multiply the values by corresponding order weights, and (4) sum up the products to obtain an overall evaluation score for a given location. For example, given a set of weighted criterion values associated with the $i$-th location, $w_{j} a_{i j}=(0.8$, $0.1,0.9,0.5)$ and a set of associated order weights $v_{j}=(0.4,0.3,0.2,0.1)$, the OWA procedure involves: reordering the weighted attribute values $\left(b_{i 1}=\right.$ $0.9, b_{i 2}=0.8, b_{i 3}=0.5$, and $\left.b_{i 4}=0.1\right)$, and combining the ordered weighted attribute values; that is, $O W A_{i}=(0.4 \times 0.9)+(0.3 \times 0.8)+(0.2 \times 0.5)+$ $(0.1 \times 0.1)=0.71$.

With different sets of order weights, one can generate a wide range of OWA operators including the three special cases of the WLC, Boolean AND and OR combinations. A set of equal order weights $\left(\frac{1}{n}, \frac{1}{n}, \ldots, \frac{1}{n}\right)$ does not affect any position in the re-ordered weighted standardized criterion values, resulting in the WLC scores; the order weights $(1.0,0.0, \ldots, 0.0)$ assign a weight of 1.0 to the highest (best) criterion value for each location, resulting in an OR-type combination; order weights $(0.0, \ldots, 0.0,1.0)$ assign a weight of 1.0 the lowest (worst) values, resulting in the Boolean AND combination (Jiang and Eastman 2000; Malczewski et al. 2003).

The behavior of the OWA operators can be described in two dimensions: the degree of ORness (or risk) and tradeoff. The measure of ORness is defined as follows (Yager 1988):

$$
\text { ORness }=\sum_{j=1}^{n}\left(\frac{n-j}{n-1}\right) v_{j}, \quad 0 \leq \text { ORness } \leq 1 .
$$

The degree of ORness indicates the position of OWA on a continuum between the AND or OR operators. It emphasizes the higher (better) values 
or the lower (worse) values in a set of attributes associated with the $i$-th alternative. There are both theoretical and empirical evidence to show that individuals (decision-makers) with optimistic (or risk-taking) attitudes tend to emphasize good properties of alternatives, while pessimistic or risk-averse decision-makers tend to focus on bad properties of alternatives (Bodily 1985; Mellers and Chang 1994).

The tradeoff is defined as follows (Jiang and Eastman 2000):

$$
\text { tradeoff }=1-\sqrt{n \sum_{j=1}^{n} \frac{\left(v_{j}-\frac{1}{n}\right)^{2}}{n-1}}, \quad 0 \leq \text { tradeoff } \leq 1 .
$$

According to Jiang and Eastman (2000) the tradeoff measure specifies the degree of compensation or substitutability between criteria. It indicates the compensation of low values on one criterion by high values on another criterion.

These two dimensions form a decision strategy space (see Fig. 1; Eastman 2000; Jiang and Eastman 2000; Rinner and Malczewski 2002). To this end, it is important to notice that the degrees of ORness/risk and tradeoff depend on the number of criteria, $n$, being included in the OWA procedure. Except for the special cases of ORness $=1.0,0.5$, and 0.0 , the greater the number of criteria, the higher the level of tradeoff that can be obtained for a given degree of ORness. For the special cases of AND, OR, and WLC operators, the measures of tradeoff and ORness are fixed irrespectively of the number of criterion maps. For $n=2$, the decision space has a triangular form. As the number of criteria increases from $n=2$ to $n \rightarrow \infty$, the decision strategy space gradually changes its shape from a triangular to a rectangular form.

\subsection{OWA in CommonGIS}

Rinner and Malczewski (2002) have presented an implementation of OWA in the CommonGIS system. Major features of this tool include its interac-

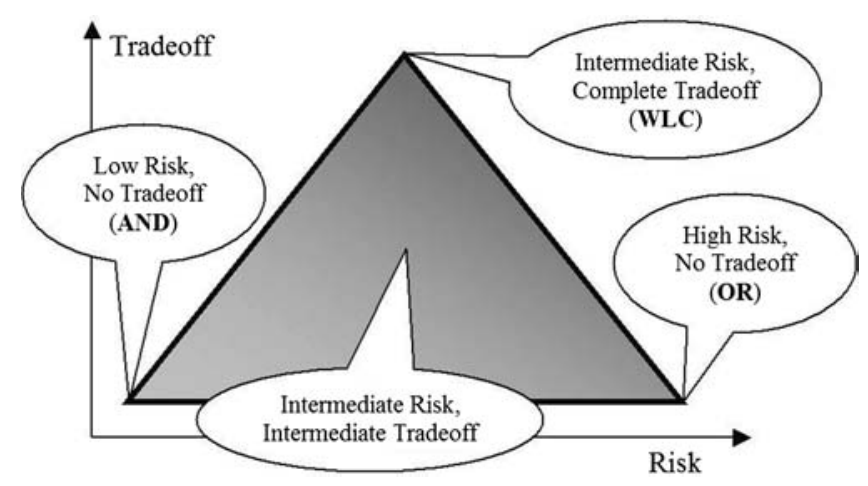

Fig. 1 The decision strategy space for $n=2$ (modified after Eastman 2000) 
tivity, Web-compliance, and vector-based approach. CommonGIS is an interactive tool for thematic cartography that supports users in exploring spatial data (Andrienko and Andrienko 1999) and includes decision support methods (Jankowski et al. 2001). The OWA method benefits from interactive features such as dynamic map updates and linked displays by allowing users to change the parameters, re-evaluate criteria and visualize new rankings. CommonGIS is a Java implementation that can run as a stand-alone application or as an applet within a Web browser. Using a vector-based approach implies that the criterion combination procedure is performed on attributes in a feature table.

The CommonGIS OWA module provides users with controls to select one of the three characteristic decision strategies (AND, OR, WLC) or to specify the order weights individually on scales between 0.0 and 1.0. The unlimited number of possible decision strategies between the special cases leads to the problem of defining appropriate order weights. The manual definition of order weights to achieve a specific behavior of the OWA operator can be a cumbersome process. Current OWA implementations in Idrisi (Eastman 1997) or CommonGIS (CommonGIS 2003) only support the "quick" selection of very few characteristic decision strategies as described above. However, when introducing OWA, Yager (1988) has suggested that the set of order weights "is a manifestation of the quantifier underlying the aggregation [combination] process".

\section{OWA extension with linguistic quantifiers}

\subsection{Linguistic quantifiers and OWA weights}

The concept of linguistic quantifiers was introduced by Zadeh (1983). The quantifiers are represented as fuzzy sets, and consequently they are also referred to as fuzzy quantifiers. The concept of fuzzy quantifiers allows for the translation of natural language specifications into formal mathematical expressions, which directly leads to the formulation of the multicriteria decision/evaluation functions (Kickert 1978; Munda 1995).

There are two generic classes of linguistic quantifiers: absolute and relative quantifiers (Zadeh 1983; Yager 1996). The former are defined as fuzzy subsets of $[0,+\infty[$. They can be used to represent linguistic statements such as about five, or more than ten. The relative quantifiers are closely related to imprecise proportions. They are defined as fuzzy subset of $[0,1]$ with proportional terms such as a few, half, many, most, etc. Here, we limit ourselves to a class of relative (proportional) quantifiers and we employ one of the most often used methods for defining a parameterized subset on the unit interval (Yager 1996): quantifier $(p)=p^{\alpha}, \alpha>0$. For a series of linguistic quantifiers that include monotonically increasing proportions of elements, we can associate the quantifiers with a value of a single parameter, $\alpha$, which indicates the degree of the inclusion. This parameter can be used to calculate a set of order weights as follows: 


$$
v_{j}=\left(\frac{j}{n}\right)^{\alpha}-\left(\frac{j-1}{n}\right)^{\alpha}, \text { for } j=1,2, \ldots, n .
$$

The order weights are generic in the sense that they are independent of particular multicriteria problems. They only depend on the number of criteria, which are being used in the combination procedure, and on the specified linguistic quantifiers, which are associated with the $\alpha$ parameter. Table 1 shows a possible matching between selected linguistic quantifiers and the $\alpha$ values. By changing the parameter one can generate different types of quantifiers and associated OWA operators between the two extreme cases of the at least one and all quantifiers (see Table 1). Once the set of order weights is known, OWA is calculated according to Eq. 1. This type of multicriteria procedure is referred to as the quantifier-guided OWA combination (Yager 1996).

\subsection{Linguistic quantifiers in CommonGIS}

We have implemented the linguistic quantifier concept in CommonGIS. The user interface components for specifying a decision strategy in the OWA dialog in CommonGIS are presented in Fig. 2. The screenshot in Fig. 2a shows the initial view of the dialog, which contains radio buttons to select one of the seven linguistic quantifiers (see Table 1). Figure $2 b$ shows additional options that were present in the first OWA implementation in CommonGIS (see Rinner and Malczewski 2002): the manual control of individual order weights, the buttons to select a default decision strategy, and the heuristics for increasing or decreasing the dimensions of the decision strategy (that is, the degree of ORness and tradeoff). In addition, a user is also offered the option to manipulate the value of $\alpha$ directly. The alpha slider is defined on a linear scale, using the decimal logarithm $(\log 10)$ of the proper $\alpha$ value. Specifically, slider values from -3.0 to 3.0 are mapped to the $\alpha$ values shown in Table 1 where 0.001 approximates zero and 1000 represents infinity.

The implementation of linguistic quantifiers in CommonGIS was added to the OWA calculation dialog (see OWACalcDlg.java in Rinner and Malczewski 2002). If a linguistic quantifier is selected, then the quantifier is mapped to the corresponding value of $\alpha$, the alpha slider is set accordingly,

Table 1 Order weights for selected linguistic quantifiers (and corresponding $\alpha$ parameters) for combining two and three criteria

\begin{tabular}{lllllllll}
\hline Linguistic quantifier & & At least one & Few & Some & Half & Many & Most & All \\
\hline$\alpha$ & & 0.001 & 0.1 & 0.5 & 1 & 2 & 10 & 1000 \\
Order weights $(n=2)$ & $v_{1}$ & 1.00 & 0.93 & 0.71 & 0.50 & 0.25 & 0.00 & 0.00 \\
& $v_{2}$ & 0.00 & 0.07 & 0.29 & 0.50 & 0.75 & 1.00 & 1.00 \\
Order weights $(n=3)$ & $v_{1}$ & 1.00 & 0.90 & 0.58 & 0.33 & 0.11 & 0.00 & 0.00 \\
& $v_{2}$ & 0.00 & 0.06 & 0.24 & 0.33 & 0.33 & 0.02 & 0.00 \\
& $v_{3}$ & 0.00 & 0.04 & 0.18 & 0.33 & 0.55 & 0.98 & 1.00 \\
Default strategy & & OR & - & - & WLC & - & - & AND \\
\hline
\end{tabular}


a
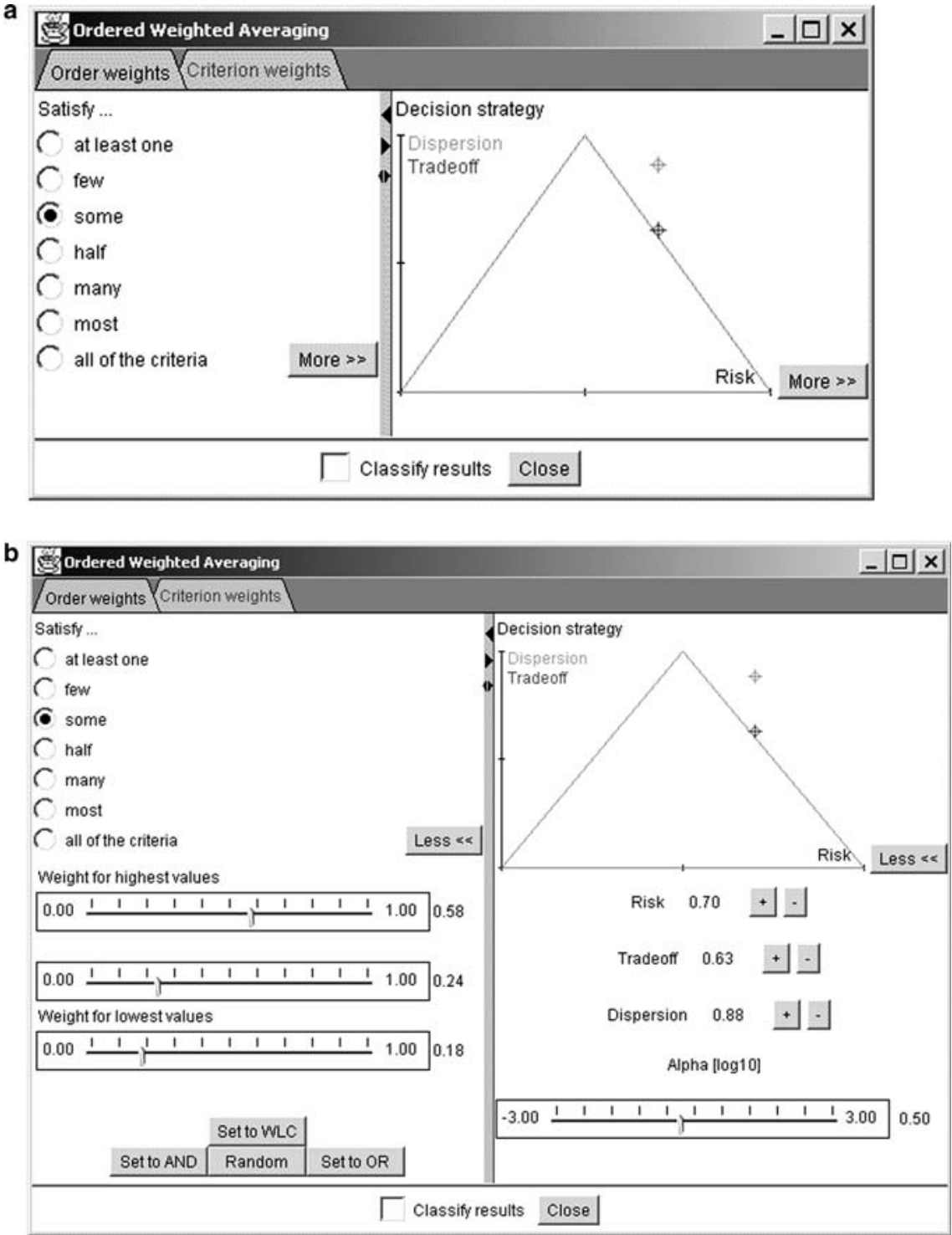

Fig. 2 User interface controls for quantifier-based OWA in CommonGIS: a initial view, b extended view

and the order weights are re-calculated and the order weight sliders adjusted to the new values. Figure 3 summarizes different options for user input as described above for the user interface screenshots. The cognitive load for performing this input can be assumed to increase from left to right. With any of the options, the display of the OWA operator's behavior in the decision strategy space is adjusted to the re-calculated order weights, and the scores 


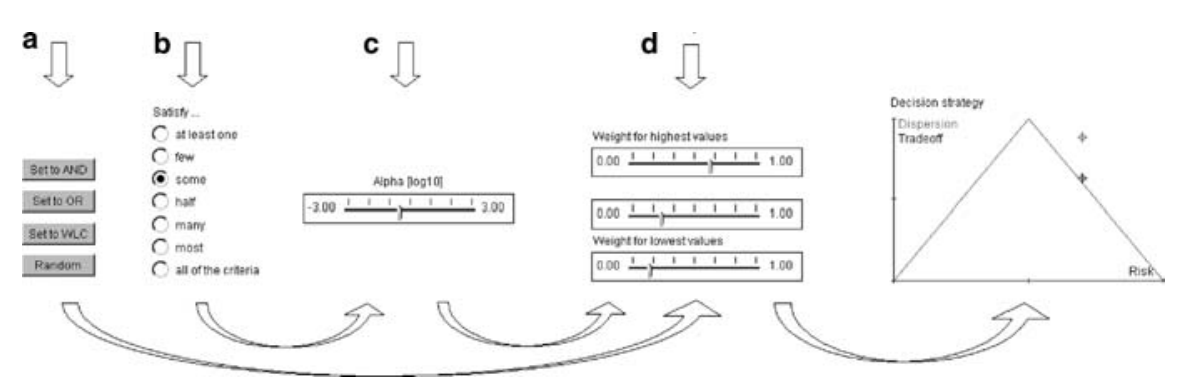

Fig. 3 Options for user input to define a decision strategy and system's update sequence: a selection of a default or random strategy, $\mathbf{b}$ selection of a linguistic quantifier, c definition of alpha parameter, $\mathbf{d}$ manual definition of order weights

and ranks of decision alternatives are re-calculated and stored in the feature table. The CommonGIS system automatically updates all maps and graphs.

\section{Illustrative example: evaluating residential quality in London, Ontario}

In 1996, the Federation of Canadian Municipalities (FCM) initiated a project on a reporting system to monitor quality of life in Canadian municipalities (FCM 1999; 2001). The project is known as the quality of life reporting system (QOLRS). At present, it involves 18 large municipalities and regional districts including the City of London, Ontario. QOLRS comprises 57 "objective" indicators categorized into eight domains: population resources, community affordability, community stress, community participation, employment, housing, health, and safety. QOLRS is a multiple-indices system; that is, the indicators have not been combined to date (CUISR 2000; FCM 2001).

There are several limitations of QOLRS. First, while QOLRS is a comprehensive and informative system, it can be criticized for lacking the integrative perspective provided by a composite measure. It is suggested that the QOLRS can be used for developing a composite measure of the quality of life (Schwartzentruber et al. 1997; CUISR 2000). Specifically, CUISR (2000) has proposed a SAW with equally weighted criteria to combine the individual indicators. The development of a composite measure raises a question concerning the set of indicators that should be included in the combination procedure. We suggest that the linguistic quantifier approach (see Sect. 3) can provide a useful insight into this problem.

Second, QOLRS represents a top-down approach for developing quality of life indicators; that is, the system is based on an experts-defined framework (Sharpe 1999). It is argued that a combination of the top-down and bottom-up approaches might provide a more meaningful framework for developing QOLRS. The bottom-up approach is characterized by greater participation of a wide variety of individuals, community groups, and stakeholders, with experts involved on more of a consultative basis (CUISR 2000). 
Third, the presumption behind QOLRS is that quality of life is best measured at the municipality scale. Consequently, the boundaries of communities are treated as coincident with the administrative boundaries of municipalities. It can be argued, however, that there are considerable intramunicipality (intra-urban) differences in the quality of life (Seidle 2002).

Finally, the capability of QOLRS can be enhanced by using GIS as a tool for visualizing and analyzing spatial patterns of individual criteria and the composite measure of the quality of life. We also suggest that Web-based spatial decision support tools, such as CommonGIS, can provide a base for a participatory or "bottom-up" approach to the development of quality of life measures (Rinner and Malczewski 2002). With that in mind, we attempt in this paper to take a direct look at the intra-urban pattern of residential quality and use the linguistic quantifier OWA and CommonGIS as tools for exploratory analysis.

Like QOLRS, this study uses a set of objective indicators for measuring quality of life. It is important to notice that there is an enormous variety of indicators that can be employed in measuring quality of life (Grayson and Young 1994; Diener and Suh 1997; Yuan et al. 1999). Grayson and Young (1994: ii) argue that "[t]here appears to be a consensus that in defining quality of life there are two fundamental sets of components and processes operating: those that relate to an internal psychological mechanism producing a sense of satisfaction or gratification with life; and those external conditions which trigger the internal mechanism." The first dimension is also defined as individual/personal quality of life, subjective well-being, or life satisfaction. With respect to the second dimension there are different terms used including urban or community quality of life, or residential quality (Rogerson et al. 1989; Can 1992; Mendes and Motizuki 2001; Massam 2002). Our case study focuses on the second dimension of the quality of life. Consequently, the set of criteria included in this case study is different from the indicators used in QOLRS. However, in the process of selecting the set of criteria we have followed the principles suggested by FCM (1999).

Table 2 shows the set of 15 criteria for evaluating residential quality in London, Ontario. The data for the evaluation criteria were available for 71 "urban" census tracts in London, Ontario. The unpopulated census tract 0035 was excluded from the analysis. For demonstration purposes we assume that equal weights have been assigned to the criteria (see CUISR 2000). Given the criterion weights, we apply the OWA procedure for seven linguistic quantifiers: all, most, many, half, some, few, and at least one.

Figure 4a shows a parallel coordinate plot of the fifteen evaluation criteria along with corresponding criterion weights (sliders for changing the values of criterion weights in the $0-1$ interval). The parallel coordinate plot allows for examining the influence of a particular criterion on the final criterion outcome (an overall evaluation score). The final criterion outcomes are shown at the bottom of the parallel coordinate panel in terms of the overall evaluation scores and the rankings of the 70 census tracts (Fig. 4a shows the evaluation scores and rankings for the half (WLC) strategy). The parallel coordinate plot is dynamically linked to the choropleth maps shown in Fig. 4b. This feature of CommonGIS allows for exploring the patterns of 
Table 2 Criteria for evaluating residential quality in London, Ontario (Source: Statistics Canada 1996; Poetz 2003)

\begin{tabular}{|c|c|c|c|}
\hline \# & Name & Description & Type \\
\hline 1 & AVE_VAL & Average value of dwelling & $\operatorname{Max}$ \\
\hline 2 & OLD_DWE & $\begin{array}{l}\% \text { of dwellings built before } 1946 \\
\text { (total number of dwellings) }\end{array}$ & Min \\
\hline 3 & NEW_DWE & $\begin{array}{l}\% \text { of dwellings built after } 1980 \\
\text { (total number of dwellings) }\end{array}$ & Max \\
\hline 4 & AVE ROO & Average number of rooms per dwelling & Max \\
\hline 5 & NON_INC & $\begin{array}{l}\% \text { of without income } \\
\text { (population } 15 \text { years and over) }\end{array}$ & Min \\
\hline 6 & LOW_INC & $\begin{array}{l}\% \text { of incidence of low income } \\
\text { (population } 15 \text { years and over) }\end{array}$ & Min \\
\hline 7 & GOV_PAY & $\begin{array}{l}\% \text { government transfer payments } \\
\text { (composition of total income) }\end{array}$ & Min \\
\hline 8 & UNE_RAT & $\begin{array}{l}\text { Unemployment rate } \\
\text { (population } 15 \text { years }+ \text { ) }\end{array}$ & Min \\
\hline 9 & UNA_IND & $\begin{array}{l}\% \text { Unattached individuals } \\
\text { (population } 15 \text { years }+ \text { ) }\end{array}$ & Min \\
\hline 10 & MOB_ONE & $\begin{array}{l}\% \text { non-movers } \\
\text { (mobility status } 1 \text { year ago) }\end{array}$ & Max \\
\hline 11 & LOW_EDU & $\begin{array}{l}\% \text { of less than grade } 9 \\
(\text { population } 15 \text { years }+ \text { ) }\end{array}$ & Min \\
\hline 12 & HIG_EDU & $\begin{array}{l}\% \text { of university education } \\
\text { (population } 15 \text { years }+ \text { ) }\end{array}$ & Max \\
\hline 13 & NON_SCH & $\begin{array}{l}\% \text { of not attending school } \\
\text { (population } 15 \text { to } 24 \text { years) }\end{array}$ & Min \\
\hline 14 & RES_BUR & $\begin{array}{l}\text { Residential burglary } \\
\text { (Relative risk ratio) }\end{array}$ & Min \\
\hline 15 & POP_DEN & $\begin{array}{l}\text { population density } \\
\left(\text { population } / \mathrm{km}^{2}\right)\end{array}$ & Min \\
\hline
\end{tabular}

residential quality in geographical space and criterion outcome space simultaneously (see Jankowski et al. 2001).

Figure $4 \mathrm{~b}$ shows choropleth maps for the results of the seven alternative decision strategies. The use of unclassed choropleths avoids artificial clustering based on a coarse classification of ranks. Each strategy is associated with a measure of tradeoff between evaluation criteria (and a value of the $\alpha$ parameter - see Figs. 2 and 3). The all (AND) strategy is referred to as the extremely pessimistic strategy. According to this strategy the lowest criterion value is assigned to each census tract as its final score. Moving from the AND operator to the half quantifier corresponds to an increasing degree of optimism as well as an increasing tradeoff between evaluation criteria. This implies that increasing (order) weights are assigned to the higher-ranking criterion values at the expense of lower-ranking criteria in a given area. The strategy associated with the half quantifier $(\alpha=0.5)$ corresponds to WLC. This strategy is characterized by a neutral attitude towards risk and a full tradeoff between criteria. Increasing the value of $\alpha$ from 0.5 (the half quantifier) to 1.0 (the at least one quantifier or the OR operator) represents an increasing degree of optimism and decreasing level of tradeoff among criteria. A comparison of the choropleth maps in Fig. 4b reveals a remarkably stable spatial pattern of residential quality in London, Ontario. Irrespective of the quantifier-defined evaluation strategy, the lowest residential 


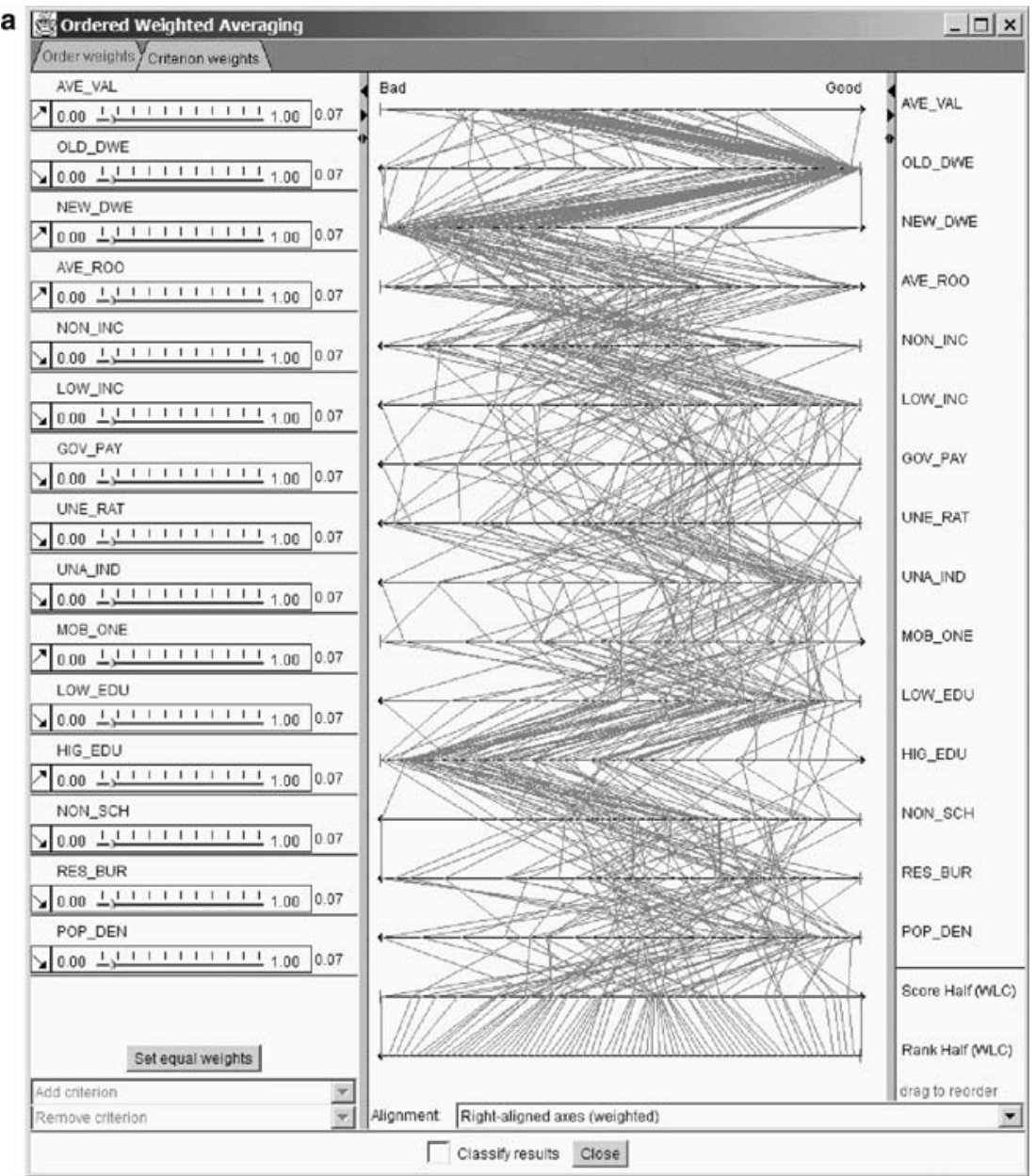

Fig. 4 a-c Evaluating residential quality in London, Ontario: a Criterion weights and parallel coordinate display of evaluation criteria, b Multiple choropleth maps of quantifier-defined decision strategies, c Parallel coordinate plot of results of the decision strategies

quality is found in the core area of the city and it tends to increase as one moves away from the core area. In addition, the poor quality residential neighborhoods tend to be more clustered in the core area along with moving from the all strategy to the at least one strategy.

Further insight into the spatial pattern of residential quality can be obtained by analyzing the pattern (visualized in geographical space) in conjunction with the data visualized in criterion outcome space. Figure $4 \mathrm{c}$ shows the evaluation scores for the 70 census tracts with respect to the quantifierdefined strategies using a parallel coordinate plot. This type of dual view of the evaluation problem provides an opportunity for exploring the sensitivity of the performance of individual neighborhoods to the changes in the 
b

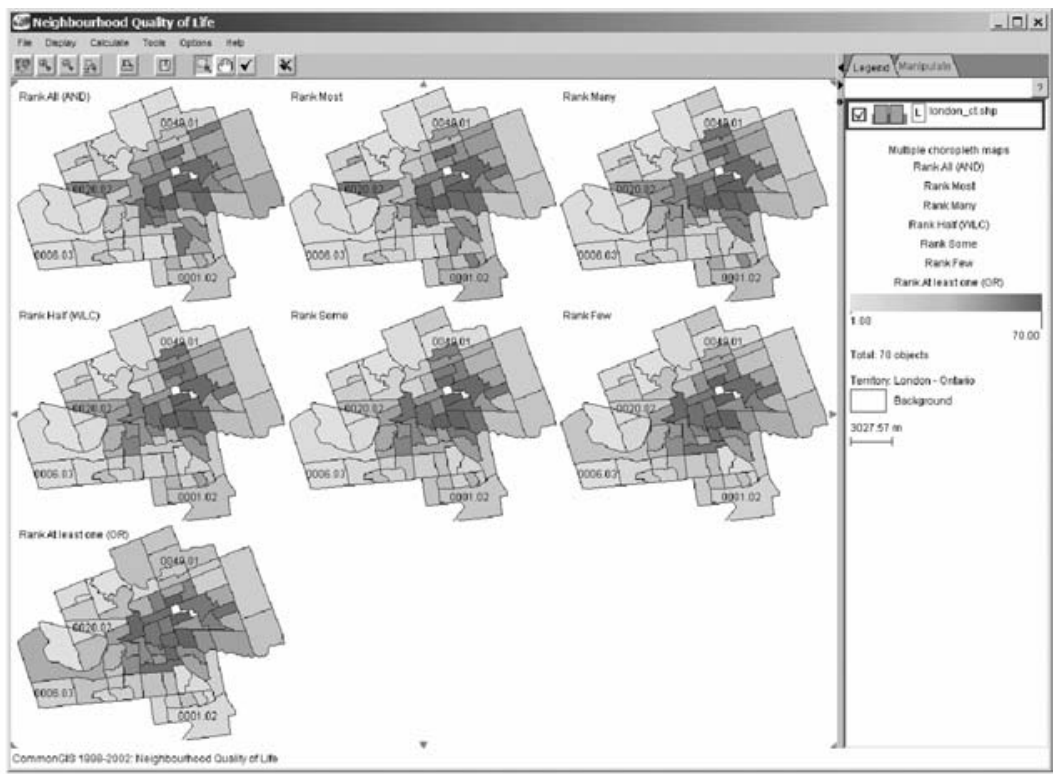

C

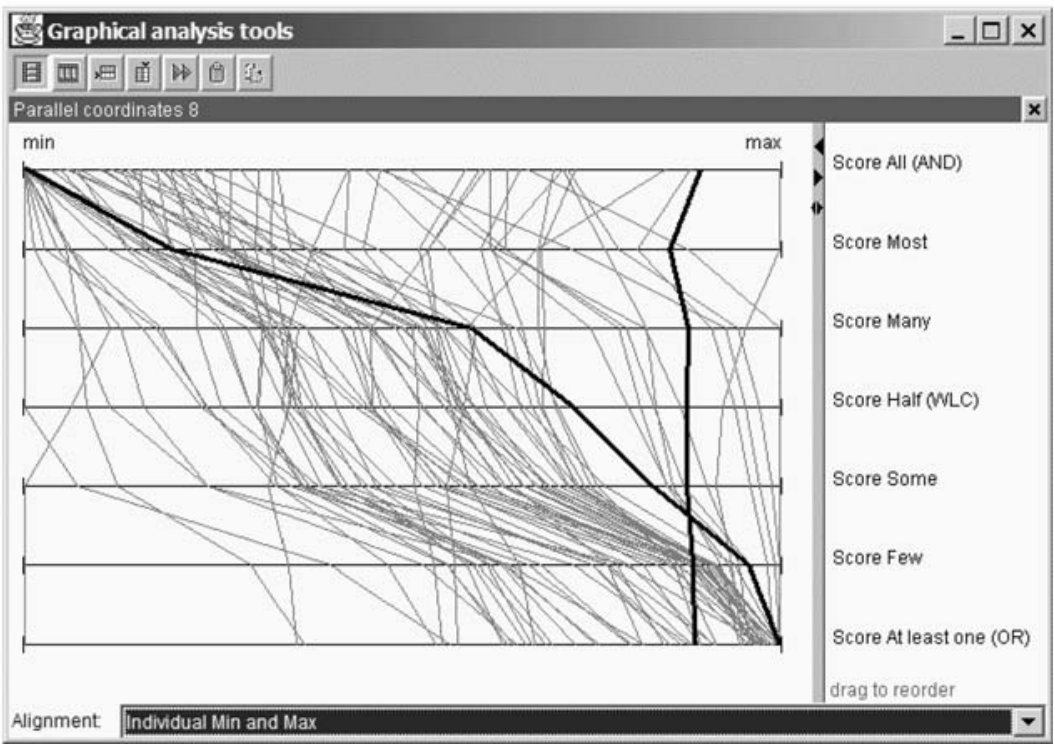

Fig. 4 (Contd.)

quantifier-defined strategies. For example, the two highlighted lines represent very different census tracts in terms of their sensitivity to changing evaluation strategies. The performance of the census tract represented by the line crossing the horizontal parallel coordinate at an angle of $\sim 90^{\circ}$ is insensitive to the changing strategies; that is, irrespectively of the strategy, the neighborhood is characterized by very similar overall evaluation scores. This 

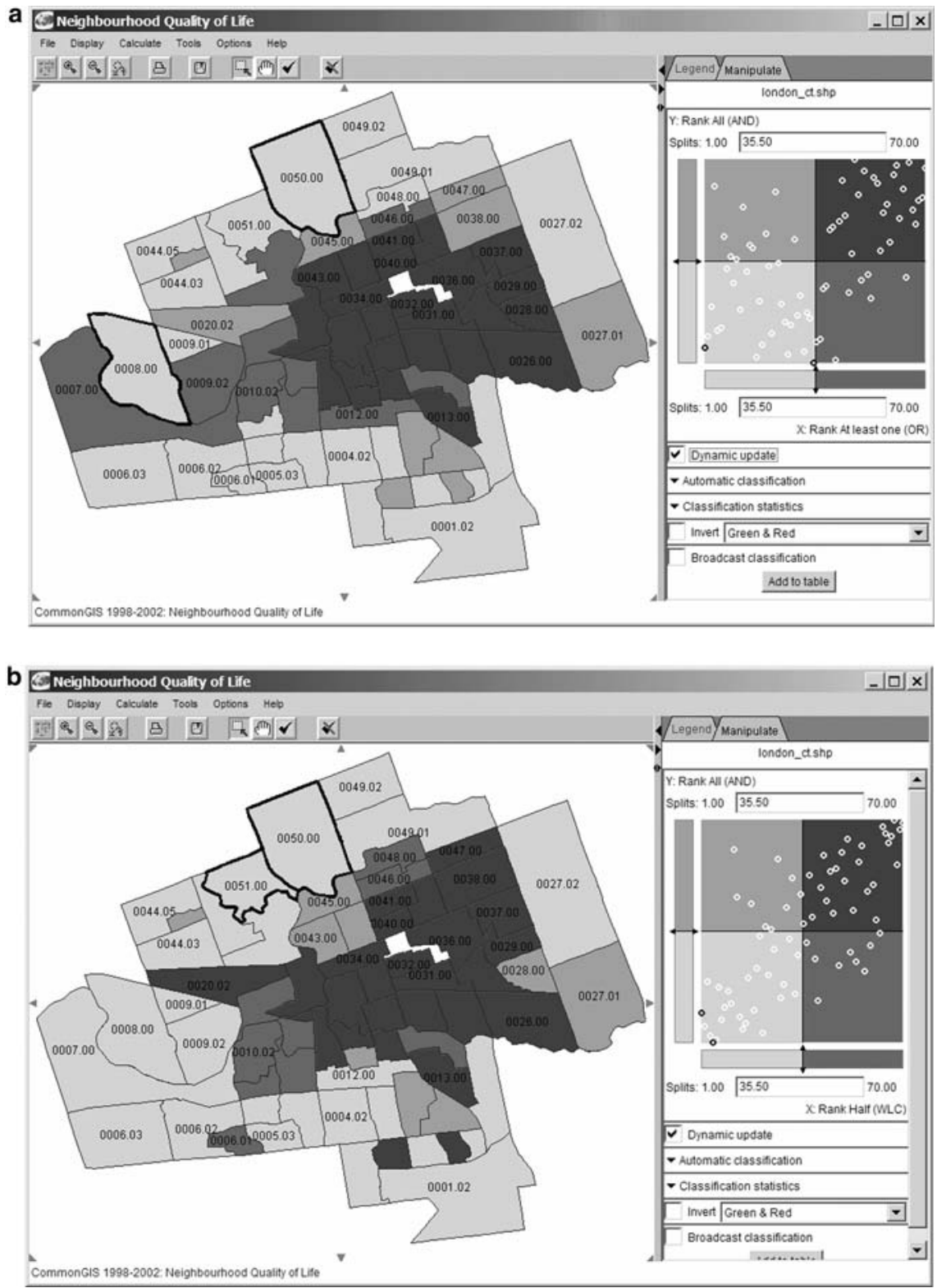

Fig. 5 a-c Cross-classification of the residential quality evaluation strategies in London, Ontario: a the all (AND) and at least one (OR) strategies, b the all (AND) and half (WLC) strategies, and $\mathbf{c}$ the half (WLC) and at least one (OR) strategies

implies that there is little variation in the standardized criterion scores. The other highlighted line exemplifies a neighborhood that is very sensitive to the changing strategies. It performs poorly for the all strategy but its performance gradually increases as one moves towards the at least one scenario. 


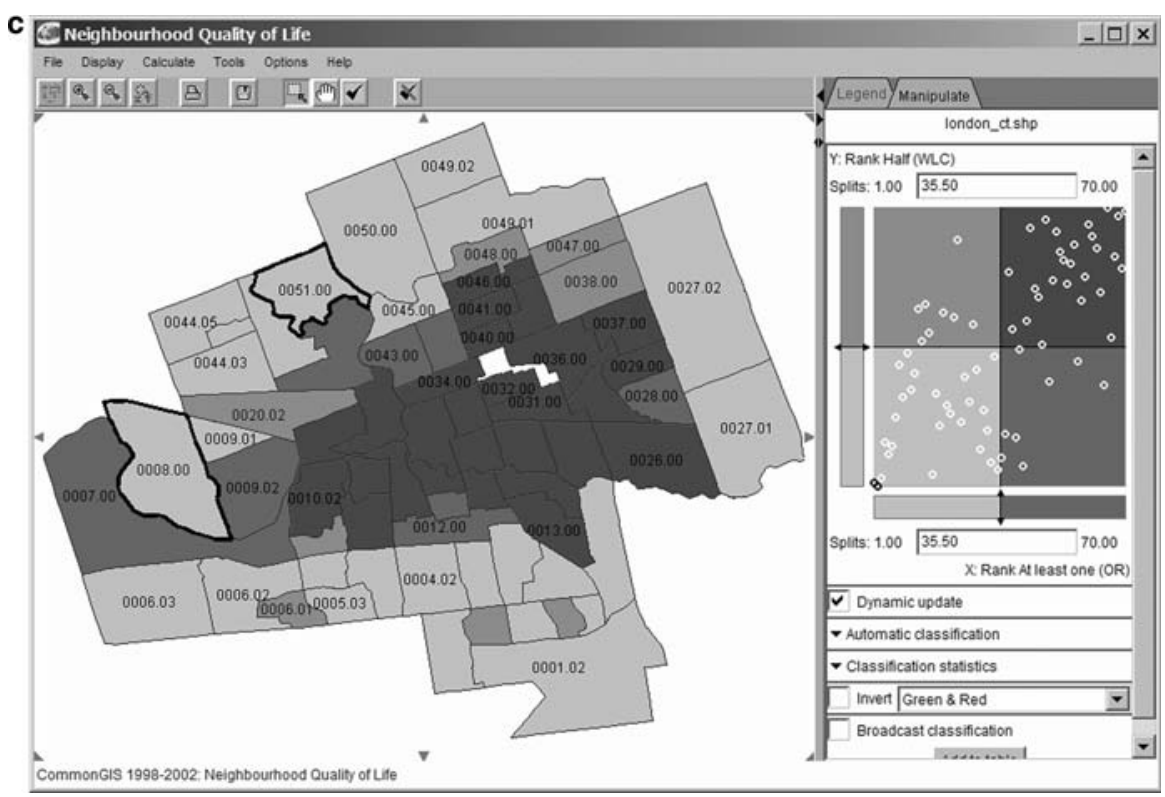

Fig. 5 (Contd.)

CommonGIS provides a number of options for classifying data sets and visualizing them in the geographical and criterion outcome spaces (Andrienko and Andrienko 1999; Jankowski et al. 2001). Figure 5 demonstrates the cross-classification option using bi-variate choropleth maps and corresponding scatterplots for the pairwise comparison of the AND, OR, and WLC strategies. An inspection of the maps indicates that there is considerable correspondence between the strategies. The highest residential quality areas tend to be located in the periphery of the city, while the poorly performing areas are mostly found in the core area of London, Ontario. The two panels in each window are dynamically linked so that an action in one panel is displayed on the other panel. For example, the highest residential quality census tracts are marked on both panels simultaneously (see Fig. 5). To this end, one should notice that two census tracts may be very similar (or different) in geographical space and very different (or similar) in criterion outcome space. The cross-classification of the AND and OR strategies shows that the best residential areas are different in both the geographic and criterion outcome spaces (see Fig. 5a). By contrast, the highest quality residential areas for the AND and WLC are similar in terms of their geographic location and the criterion outcomes (see Fig. 5b). Finally, the OR and WLC operators result in the best residential quality areas which are very similar in the criterion space while they are different in the geographic space (see Fig. 5c). This type of dynamically linked displays provides an important insight into the nature of the evaluation problem. There is some evidence to suggest (Church et al. 1992; Jankowski et al. 2001) that people usually have strong ideas about the geographic space and when shown two alternatives 
that have similar values in the criterion space, they will often have strong preferences for one alternative over the other because of their locations in geographic space.

Another type of visual technique for exploring the results of the linguistic quantifier-guided OWA is to use multiple choropleth maps in conjunction with dot plots representing the distribution of final ranks. Figure 6 shows the highest residential quality areas for the seven strategies by the lightest grey shades on the choropleth maps. In addition, the boundaries of the highest residential quality neighborhoods are marked according to the seven strategies (see Fig. 5). The unclassed choropleth mapping method in CommonGIS also allows us to interactively set a reference value (e.g. rank $=10)$ to split the choropleth color scheme (not shown on grey scale print). The top ten residential areas tend to be located in the north and west sections of the city. These areas include the three highest residential quality neighborhoods of London, Ontario.

\section{Conclusions}

In this paper, we have presented an extension of an existing GIS-based approach to ordered weighted averaging with linguistic quantifiers. The quantifier-guided OWA procedures are most useful in situations involving a large number of evaluation criteria. We have argued that for a large set of evaluation criteria it may be difficult or even impossible to quantitatively specify the relationships between criteria. The linguistic quantifier-based approach has been demonstrated to simplify the definition of the relationships. It has also provided us with a simplified way of defining the parameters associated with the OWA combination procedures.

The paper has emphasized the importance of using the quantifier-guided OWA in conjunction with an exploratory multicriteria analysis. Interactive thematic mapping tools such as CommonGIS allow for exploring decision or evaluation problems in the geographic space and the criterion outcome space, simultaneously. Using an example of residential quality evaluation we have demonstrated that the criterion outcome space may not capture some vital geographical components. Some neighborhoods may be very similar in the criterion space but they may be very different when analyzed in the geographic space, and vice versa. An interactive/exploratory analysis of the geographic pattern and the distribution of alternatives in criterion space opens an opportunity for exploring the nature of a decision problem that, consequently, may provide new insights into the relationships between evaluation criteria and between decision alternatives.

This research has demonstrated that there is a core-periphery pattern of residential quality in London, Ontario. Specifically, the disadvantaged neighborhoods tend to be concentrated in the core area of the city, while the high quality neighborhoods tend to be located in the peripheral areas. The core-periphery pattern is remarkably stable; that is, irrespective of the quantifier-defined evaluation strategy, the lowest residential quality is found in the core area of the city and residential quality tends to increase as one 


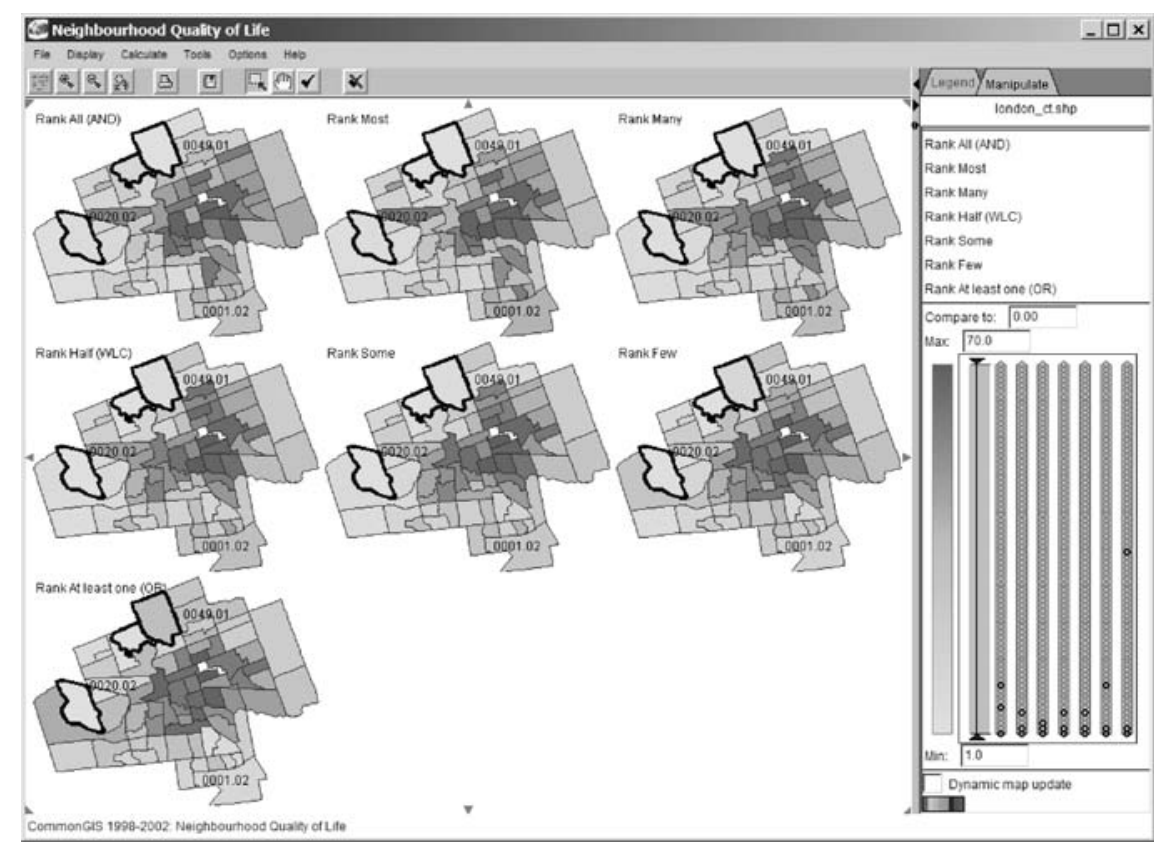

Fig. 6 Ranking of census tracts in London, Ontario, for different evaluation strategies. Lighter shades of grey correspond to higher residential quality. The three best census tracts from Fig. 5 are outlined in black

moves away from the core area. These results provide support for growing concerns about the increasing spatial concentration of the "distressed neighborhoods" within Canadian cities (Bradford 2002; Seidle 2002). Furthermore, Seidle (2002) suggests that public participation in the discussions and program development around the issues of spatial concentration of "distressed neighborhoods" may lead to more effective approach to the evaluation of residential quality. The Web-enabled GIS has the potential to contribute to the bottom-up approach by providing methods and tools that can aid in participatory evaluation of residential quality.

More research into the usability and effectiveness of exploratory multicriteria evaluation in GIS is needed and we plan a study with a user group to test the proposed method in a real-world setting. We also want to offer a larger choice of decision support methods in conjunction with the interactive cartographic and graphic features of the CommonGIS platform to allow researchers to further compare spatial and non-spatial decision outcomes, and to assist decision makers in finding the method that best fits their needs.

\section{References}

Andrienko GL, Andrienko NV (1999) Interactive maps for visual data exploration. Int J Geogr Inf Syst 13(4):355-374 
Andrienko N, Andrienko G (2001) Intelligent support for geographic data analysis and decision making in the Web. J Geogr Inf Decis An 5(2):115-128

Andrienko N, Andrienko G (2003) Informed spatial decisions through coordinated views. Inform Vis 2(4):270-285

Banai R (1993) Fuzziness in geographic information systems: contributions from the analytic hierarchy process. Int J Geogr Inf Syst 7(4):315-329

Bayless M, Bayless S (1982) Current quality of life indicators: some theoretical and methodological concerns. Am J Econ Sociol 41(4):421-437

Bodily SE (1985) Modern decision making: a guide to modeling with decision support systems. McGraw-Hill Book Company, New York

Bradford N (2002) Why cities matter: policy research perspectives for Canada. CPRN Discussion Paper No. F23, Canadian Policy Research Networks, Ottawa, pp 97

Can A (1992) Residential quality assessment: alternative approaches using GIS. Ann Regional Sci 23(1):97-110

Carver SJ (1991) Integrating multi-criteria evaluation with geographical information systems. Int J Geogr Inf Syst 5(3):321-339

Carver SJ (1999) Developing Web-based GIS/MCE: Improving access to data and spatial decision support tools. In: Thill J-C (ed) Multicriteria decision-making and analysis: A geographic information sciences approach. Ashgate, New York, pp 49-76

Church RL, Loban SR, Lombard K (1992) An interface for exploring spatial alternatives for a corridor location problem. Comput Geosci 8(10):1095-1105

CommonGIS (2003) CommonGIS - Thematic mapping for everyone... everywhere, http:// www.commongis.com [accessed:18 March 2004]

CUISR (Community-University Institute for Social Research) (2000) An evaluation of The Federation of Canadian Municipalities Quality of Life Reporting System, Community-University Institute for Social Research, University of Saskatchewan, Saskatoon. pp 31

Diener E, Suh E (1997) Measuring quality of life: economic, social, and subjective indicators. Soc Indic Res 40:189-216

Eastman JR (1997) IDRISI for Windows, Version 2.0: Tutorial exercises. Graduate School of Geography, Clark University, Worcester

Eastman JR (2000) Decision strategies in GIS. Column in directions magazine, dated December 13, 2000. Available online at http://www.directionsmag.com/ columns.php?column_id = 38 [accessed 18 March 2004]

FCM (Federation of Canadian Municipalities) (1999) The FCM Quality of Life Reporting System: Quality of Life in Canadian Communities. Federation of Canadian Municipalities, Ottawa, pp 93

FCM (Federation of Canadian Municipalities) (2001) The FCM quality of life reporting system: quality of life in Canadian communities, second report. Federation of Canadian Municipalities, Ottawa, pp 115

Grayson L, Young K (1994) Quality of life in cities: an overview and guide to the literature, The British Library, London

Heywood I, Cornelius S, Carver S (2002) An introduction to geographical information Systems. Prentice Hall, Harlow

Heywood I, Oliver J, Tomlinson S (1995) Building an exploratory multi-criteria modeling environment for spatial decision support. In: Fisher P (ed), Innovations in GIS 2, Taylor \& Francis, Bristol, pp 127-136

Hogarth R (1983) Judgment and choice. Wiley, Chichester

Hwang C-L, Yoon K (1981) Multiple attribute decision making: methods and applications. Springer, Berlin Heidelberg New York

Jankowski P (1995) Integrating geographical information systems and multiple criteria decision making methods. Int J Geogr Inf Syst 9(3):251-273

Jankowski P, Andrienko N, Andrienko G (2001) Map-centered exploratory approach to multiple criteria spatial decision making. Int J Geogr Inf Sci 15(2):101-127

Janssen R, Rietveld P (1990) Multicriteria analysis and geographical information systems: An application to agricultural land use in the Netherlands. In: Scholten HJ, Stillwell $\mathrm{JCH}$ (eds) Geographical information systems for urban and regional planning. Kluwer, Dordrecht, pp 129-139

Jiang H, Eastman JR (2000) Application of fuzzy measures in multi-criteria evaluation in GIS. Int J Geogr Inf Syst 14(2):173-184 
Joerin F, Thériault M, Musy A (2001) Using GIS and outranking multicriteria analysis for landuse suitability assessment. Int J Geogr Inf Sci 15(2):153-174

Kickert WJM (1978) Fuzzy theories of decision-making. Martinus Nijhoff, Leiden

Laaribi A, Chevallier JJ, Martel JM (1996) A spatial decision aid: a multicriterion evaluation approach. Comput, Environ Urban 20(6):351-366

Malczewski J (1999) GIS and multicriteria decision analysis. Wiley, New York

Malczewski J (2000) On the use of weighted liner combination method in raster GIS: common and best practice approaches. Trans GIS 4(1):5-22

Malczewski J, Chapman T, Flegel C, Walters D, Shrubsole D, Healy M (2003) GISmulticriteria evaluation with ordered weighted averaging (OWA): case study of developing watershed management strategies. Environ Plan A 35(10):1769-1784

Massam BH (1993) The right place: shared responsibility and the location of public facilities. Longman Scientific \& Technical, Harlow

Massam BH (2002) Quality of life: public planning and private living. Prog Plann 58(3):141-227

Mellers B, Chang S (1994) Representations of risk judgments. Organ Behav Hum Dec 52(7):167-184

Mendes JFG, Motizuki WS (2001) Urban quality of life evaluation scenarios: the case of São Carlos in Brazil. CTBUH Rev 1(2):1-10

Munda G (1995) Multicriteria evaluation in a fuzzy environment: theory and applications in ecological economics. Physica-Verlag, Heidelberg

Nijkamp P, Leitner H, Wrigley N (1985) Measuring the unmeasurable. Martinus Nijhoff Publishers, Dordrecht

O'Sullivan D, Unwin DJ (2003) Geogr Inf Anal. Wiley, Hoboken

Pereira JMC, Duckstein L (1993) A multiple criteria decision-making approach to GISbased land suitability evaluation. Int J Geogr Inf Syst 7(5):407-424

Poetz A (2003) Spatial patterns of residential Burglaries in London, Ontario. Unpublished B.A. Thesis, University of Western Ontario, London, Ontario

Raphael D (1996) Defining quality of life. Eleven debates concerning its measurement. In: Renwick R, Brown I, Nagler M (eds) Quality of life in health promotion and rehabilitation: conceptual approaches, issues and applications. Sage Publications, London

Rinner C (2003) Web-based spatial decision support: status and research directions. J Geogr Inf Decis Anal 7(1):14-31

Rinner C, Malczewski J (2002) Web-enabled spatial decision analysis using ordered weighted averaging (OWA). J Geogr Syst 4(4):385-403

Rogerson RJ, Findlay AM, Morris AC, Coobes MG (1989) Indicators of quality of life: Some methodological issues. Environ Plann A 21:1655-1666

Schwartzentruber B, Baker T, Shookner M (1997) Quality of life index project methodology report. A partnership project of the Ontario Social Development Council, the Social Planning Network of Ontario, the Centre for Health Promotion at the University of Toronto and the Ontario Healthy Communities Coalition

Seidle FL (2002) The Federal Role in Canada's Cities: overview of issues and proposed actions. Discussion Paper F27, Canadian Policy Research Networks, Ottawa, pp 27

Sharpe A (1999) A survey of indicators of economic and social well-being. Canadian Policy Research Networks, Ottawa, pp 73

Starr MK, Zeleny M (1977) MCDM - State and future of the arts. In: Starr MK, Zeleny M (eds) Multiple criteria decision making, North-Holland, Amsterdam, pp 5-29

Statistics Canada (1996) Canada - Census-1996. Data located at Internet Data Library System, The University of Western Ontario

Thill J-C (1999) Multicriteria decision-making and analysis: a geographic information sciences approach. Ashgate, New York

Voß H, Andrienko N, Andrienko G (2000) CommonGIS - common access to geographically referenced data. ERCIM News 41: 44-46. Available online at http://www. ercim.org/publication/Ercim_News/enw41/voss.html [accessed 18 March 2004]

Yager RR (1988) On ordered weighted averaging aggregation operators in multi-criteria decision making. IEEE Trans Syst Man Cybern 18(1):183-190

Yager RR (1996) Quantifier guided aggregation using OWA operators. Int J Intell Syst 11:49-73

Yager RR, Kacprzyk J (eds) (1997) The ordered weighted averaging operators: theory and applications. Kluwer, Dordrecht 
Yuan LL, Yuen B, Low C (eds) (1999) Urban quality of life: critical issues and options. School of Building and Real Estate, University of Singapore, Singapore

Zadeh LA (1983) A computational approach to fuzzy quantifiers in natural languages. Comp Math Appl 9:149-184

Zhu X, Dale AP (2001) JavaAHP: A Web-based decision analysis tool for natural resource and environmental management. Environ Modell Softw 16(3):251-262 